\title{
Transmission and wind investment in a deregulated electricity industry
}

Maurovich-Horvat, Lajos; Boomsma, Trine Krogh; Siddiqui, Afzal S.

Published in:

I E E E Transactions on Power Systems

DOI:

10.1109/TPWRS.2014.2367107

Publication date:

2015

Document version

Publisher's PDF, also known as Version of record

Citation for published version (APA):

Maurovich-Horvat, L., Boomsma, T. K., \& Siddiqui, A. S. (2015). Transmission and wind investment in a deregulated electricity industry. I E E E Transactions on Power Systems, 30(3), 1633-1643.

https://doi.org/10.1109/TPWRS.2014.2367107 


\title{
Transmission and Wind Investment in a Deregulated Electricity Industry
}

\author{
Lajos Maurovich-Horvat, Trine K. Boomsma, and Afzal S. Siddiqui
}

\begin{abstract}
Adoption of dispersed renewable energy technologies requires transmission network expansion. Besides the transmission system operator (TSO), restructuring of electricity industries has introduced a merchant investor (MI), who earns congestion rents from constructing new lines. We compare these two market designs via a stochastic bi-level programming model that has either the MI or the TSO making transmission investment decisions at the upper level and power producers determining generation investment and operation at the lower level while facing wind power variability. We find that social welfare is always higher under the TSO because the MI has incentive to boost congestion rents by restricting capacities of transmission lines. Such strategic behavior also limits investment in wind power by producers. However, regardless of the market design (MI or TSO), when producers behave à la Cournot, a higher proportion of energy is produced by wind. In effect, withholding of generation capacity by producers prompts more transmission investment since the TSO aims to increase welfare by subsidizing wind and the MI creates more flow to maximize profit.
\end{abstract}

Index Terms-Market design, mathematical programming with equilibrium constraints (MPEC), transmission, wind power.

\section{NOMENCLATURE}

Indices and Sets:

$i \in \mathcal{I} \quad$ Power producers.

$\mathcal{I}^{\mathrm{c}} \subseteq \mathcal{I} \quad$ Conventional power producers.

$\mathcal{I}^{\mathrm{w}} \subseteq \mathcal{I} \quad$ Wind power producers.

$n \in \mathcal{N} \quad$ Grid nodes.

$n_{\ell}^{+} \quad$ Node index for starting node of line $\ell$.

$n_{\ell}^{-} \quad$ Node index for ending node of line $\ell$.

$\ell \in \mathcal{L} \quad$ Transmission lines.

Manuscript received February 10, 2014; revised July 19, 2014 and September 24, 2014; accepted November 02, 2014. Date of publication November 20, 2014; date of current version April 16, 2015. The work of L. Maurovich-Horvat was supported by the UCL Centre in Financial Computing. The work of T. K. Boomsma was supported by the ENSYMORA project funded by the Danish Council for Strategic Research . The work of Boomsma and Siddiqui was supported by the RISKY-RES project funded by the Research Council of Norway . Paper no. TPWRS-001940-2014.

L. Maurovich-Horvat is with the Department of Statistical Science, University College London, London, U.K. (e-mail: 1.horvat@ucl.ac.uk).

T. K. Boomsma is with the Department of Mathematical Sciences, University of Copenhagen, Copenhagen, Denmark (e-mail: trine@math.ku.dk).

A. S. Siddiqui is with the Department of Computer and Systems Sciences, Stockholm University, Stockholm, Sweden, and also with the Department of Statistical Science, University College London, London, U.K. (e-mail: afzal. siddiqui@ucl.ac.uk).

Color versions of one or more of the figures in this paper are available online at http://ieeexplore.ieee.org.

Digital Object Identifier 10.1109/TPWRS.2014.2367107 $j \in \mathcal{J}_{\ell} \quad$ Discrete capacity level of transmission investment on line $\ell$ (including the existing level, $\left.j_{0}\right)$.

$\mathcal{L}_{n}^{+} \quad$ Set of lines starting at node $n$.

$\mathcal{L}_{n}^{-} \quad$ Set of lines ending at node $n$.

$t \in \mathcal{T} \quad$ Time periods.

$s \in \mathcal{S} \quad$ Scenarios.

Parameters:

$C_{\ell, j}^{a r c} \quad$ Amortized expansion cost (including for the existing level, $\left.C_{\ell, j_{0}}^{a r c}=0\right)$ of line $\ell$ with capacity level $j(€ / \mathrm{MW})$.

$K_{\ell, j}^{a r c} \quad$ Transmission capacity (including for the existing level, $K_{\ell, j_{0}}^{a r c}$ ) of line $\ell$ with capacity level $j$ (MW).

$B_{\ell, j} \quad$ Network susceptance of line $\ell$ with capacity level $j(1 / \Omega)$.

$S_{n} \in\{0,1\} \quad$ Dummy parameter for slack node $(-)$.

$A_{n}^{i n t} \quad$ Intercept of the inverse demand curve at node $n(€ / \mathrm{MW})$.

$A_{n}^{s l p} \quad$ Slope of the inverse demand curve at node $n\left(€ / \mathrm{MW}^{2}\right)$.

$\Theta_{i^{\prime}, i, n} \quad$ Conjectured response of producer $i^{\prime}$ on the change in sales by producer $i$ at node $n(-)$.

$C_{n, i}^{i n v} \quad$ Amortized investment cost of producer $i$ at node $n(€ / \mathrm{MW})$.

$C_{n, i}^{\text {prod }} \quad$ Generation cost of producer $i$ at node $n$ (€/MW).

$K_{n, i}^{\text {prod }} \quad$ Initial generation capacity of producer $i$ at node $n$ (MW).

$E_{n, t, s}$ Availability factor for wind generation at node $n$ in period $t$ for scenario $s(-)$.

$P_{s} \quad$ Probability of scenario $s(-)$.

$R \quad$ Renewable portfolio standard (RPS) requirement $(\%)$.

\begin{tabular}{ll}
\multicolumn{2}{c}{ Primal Variables: } \\
$d_{n, t, s}$ & Voltage angle at node $n$ in period $t$ for \\
& scenario $s(\mathrm{rad})$. \\
$u_{\ell, j}$ & Transmission investment in capacity level $j$ \\
& for line $\ell(\mathrm{MW})$. \\
$f_{\ell, j, t, s}$ & Realized flow on line $\ell$ at capacity level $j$ in \\
& period $t$ for scenario $s(\mathrm{MW})$. \\
$\hat{f}_{\ell, t, s}$ & Realized flow on line $\ell$ in period $t$ for scenario \\
& $s$ (MW). \\
$g_{n, i}^{i n v}$ & Generation capacity investment at node $n$ by \\
& producer $i(\mathrm{MW})$.
\end{tabular}




$$
\begin{array}{ll}
q_{n, i, t, s}^{\text {prod }} & \text { Generation at node } n \text { by producer } i \text { in period } \\
q_{n, i, t, s}^{\text {sell }} & t \text { for scenario } s(\mathrm{MW}) . \\
& \text { Sales at node } n \text { by producer } i \text { in period } t \text { for } \\
p_{n, t, s} & \text { scenario } s(\mathrm{MW}) . \\
& \text { Electricity price at node } n \text { in period } t \text { for } \\
& \text { scenario } s(€ / \mathrm{MW}) .
\end{array}
$$

Dual Variables:

$$
\begin{array}{ll}
\mu_{\ell, j, t, s}^{+}, \mu_{\ell, j, t, s}^{-} & \begin{array}{l}
\text { Shadow price on capacity for transmission } \\
\text { line } \ell \text { at capacity level } j \text { in period } t \text { for }
\end{array} \\
& \text { Scenario } s(€ / \mathrm{MW}) \text {. } \\
\psi_{\ell, j, t, s} & \text { Shadow price on electricity flow on line } \ell \\
& \text { at capacity level } j \text { in period } t \text { for scenario } s \\
& (€ / \mathrm{MW}) . \\
\kappa_{\ell, t, s} & \text { Shadow price on electricity flow on line } \ell \text { in } \\
& \text { period } t \text { for scenario } s(€ / \mathrm{MW}) . \\
\tau_{n, t, s} & \text { Congestion fee at node } n \text { in period } t \text { for } \\
& \text { scenario } s(€ / \mathrm{MW}) . \\
\xi_{n, t, s} & \text { Dual for slack node } n \text { in period } t \text { for scenario } \\
\lambda_{n, i, t, s}^{\mathrm{c}}, & s(-) . \\
\lambda_{n, i, t, s}^{\mathrm{w}} & \text { Shadow price on generation capacity at node } \\
& n \text { for producer } i \text { in period } t \text { for scenario } s \\
\theta_{i, t, s} & (€ / \mathrm{MW}) . \\
& \text { Shadow price on energy balance for producer } \\
v_{t, s} & i \text { in period } t \text { for scenario } s(€ / \mathrm{MW}) . \\
& \text { Renewable energy certificate (REC) price in } \\
& \text { period } t \text { for scenario } s(€ / \mathrm{MW}) .
\end{array}
$$

\section{INTRODUCTION}

\section{A. Background}

$\mathbf{R}$ ESTRUCTURING of the electric power industry was precipitated by the belief that the regulated paradigm would not meet growing demand efficiently [1]. As the functions of the industry such as generation, distribution, and retailing could be handled together by an investor-owned utility (IOU) with transmission planning and reliability under the auspices of a system operator, there was little incentive to develop new technologies for the market when profits were regulated. At the same time, since a single IOU operated in each area and prices were merely set administratively, there was no need for either risk management or strategic analysis. Although a plethora of post-restructuring market designs have emerged [2], they have generally required incumbent IOUs to divest their generation assets with transmission and distribution remaining regulated. Consequently, these reforms have introduced endogenous price formation and imperfect competition, which necessitate a strategic view of decision making [3]. Moreover, market-driven transmission investment has been proposed by the US FERC's July 2002 Standard Market Design (SMD) and the EU's Regulation EC 1228/2003 [4].

Recently, sustainability issues have entered the policy debate. Several governments are committed to $\mathrm{CO}_{2}$ emissions targets in order to mitigate the effects of climate change, e.g., the EU's aim of 20-20-20 by 2020 [5]. The policymaking dilemma is to forge a delicate balance between achieving the targets while not interfering with industry. Ironically, relative to the centralized paradigm, policymakers have ceded more control to industry while simultaneously having set loftier goals in terms of economic efficiency and environmental sustainability. Since much of the transition to a sustainable energy system will rely on wind as a lynchpin technology, aspects of wind such as intermittency, non-dispatchability, and remoteness mean that policymakers will need to consider concomitant transmission expansion when devising measures to encourage wind investment [6]. Consequently, policymakers require a deeper understanding of how market designs interact with strategic behavior in delivering outcomes.

\section{B. Literature Review}

Under regulation, conventional least-cost methods could be employed to determine optimal transmission and generation investment [7]. However, with deregulation, transmission and generation investment are made by separate entities with distinct and often conflicting incentives. For example, regulated transmission system operators (TSOs) seek to maximize social welfare, while power companies are interested in profit maximization. In order to handle such game-theoretic interactions, complementarity modelling has been proposed to find Nash equilibria, i.e., solutions from which no agent has a unilateral incentive to deviate [8], [9]. Furthermore, complementarity modelling is amenable for analyzing strategic behavior in deregulated power industries due to its accommodation of physical features of the power system [10].

Bi-level problems are particularly relevant for policy analysis of the strategic interactions that arise when a dominant (leader) agent influences equilibrium prices by anticipating the decisions of followers at the lower level. Effectively, the leader's optimization problem is constrained by a set of optimization problems and equilibrium constraints at the lower level. If each lower-level problem is convex, then it may be replaced by its Karush-Kuhn-Tucker (KKT) conditions, thereby re-formulating the bi-level problem as a mathematical program with equilibrium constraints (MPEC). As [11] demonstrate in addressing the optimal offering strategy of a dominant power company, the endogeneity in the objective function of an MPEC may be resolved by using strong duality to render the problem as a mixed-integer linear program (MILP) and to treat complementarity conditions via disjunctive constraints [12]. Bi-linear expansion may alternatively resolve the endogeneity in the MPEC's objective function [13]. Closer to our effort are [14] with a welfare-maximizing TSO at the upper level making transmission investment constrained by market clearing at the lower levels and [15] with a cost-minimizing TSO at the upper level making both transmission and wind investment decisions constrained by market-clearing decisions of producers at the lower level(s). In contrast to work that assumes a fixed transmission network [16], [15] illustrates the need to consider transmission and wind jointly.

Still within a bi-level framework, [17] uses the framework of [18] to investigate a two-stage duopoly in which producers make investment decisions in the first stage and operational ones in the second stage. The resulting (closed-loop) bi-level equilibrium problem with equilibrium constraints (EPEC) yields the same result as an open-loop mixed-complementarity problem (MCP) for any conjectural variation in the spot market as long 
as there is a single load period and the spot market is at least as competitive as in the Cournot case. This justifies a singlelevel approximation of the producers' bi-level problem. Such an equivalence may still hold when there are multiple load periods as demonstrated by an example from [17]. However, at the same time, [17] presents a counter-example in which the installed capacity is actually lower in the closed-loop (EPEC) model relative to the open-loop (MCP) model when spot markets are closer to being perfectly competitive, thereby indicating that open-loop results may not always generalize for multiple time periods. Moving on to a tri-level model, [19] and [20] have a welfare-maximizing TSO at the upper level making transmission investments, producers at the middle level making generation capacity investments, and market clearing at the lower level. Thus, this is a more complicated problem than even an EPEC, and neither [19] nor [20] attempts to solve it directly. Rather, they compare pre-determined transmission investment proposals from the perspective of various planners. In contrast to [19], [20] focuses on market power by the producers and notes that diverging objectives for the TSO may lead to politically infeasible investment plans.

Although transmission expansion has largely remained under the control of regulated TSOs, market-based models for transmission investment have been proposed in both the UK and the US. For example, [21] posits a role for a merchant investor (MI) who would build new transmission lines motivated by the collection of congestion rents between grid nodes. However, the efficient outcomes hypothesized by [21] under the MI are subverted if market power exists [22]. In discussing the landscape for merchant transmission investment in Europe, [23] notes that financial transmission rights (FTRs) would be beneficial for dealing with externalities and providing hedging capabilities for investors. Yet, empirical analyses of markets for FTRs in the USA have shown inefficiencies, e.g., divergent forward and spot prices for congestion rents, to exist in their operations, especially in congested regions [24].

\section{Research Objectives and Contribution}

Given this background, we aim to gain policy insights into market design by analyzing transmission and wind investment by distinct agents reflecting strategic behavior: at the upper level, we posit that a TSO or an MI invests in new transmission lines (acting as a Stackelberg leader), while at the lower level, profit-maximizing conventional and wind producers make investment and operational decisions with transmission flows governed by the relevant grid owner. In contrast to [15], we allow for market power at the lower level and find that this specification of industry (as behaving either perfectly competitively or à la Cournot) affects both lower- and upper-level decisions. Moreover, unlike the extant literature, we investigate the implications of transmission investment made by an MI in a bi-level model. Finally, we explore the impact of a policy measure such as the renewable portfolio standard (RPS) [25]. A cap-and-trade mechanism for emissions could also be investigated using our approach [26].

We demonstrate that results are largely intuitive if producers at the lower level are price takers: generation capacity is added by the least costly producers, the conventional producer at the node with the highest demand does not face competition, and power flows from the less costly wind producer to the node with more capital-intensive (wind) producers. The impact of having the TSO or the MI at the upper level affects mainly the magnitudes of the outcomes and not their fundamental compositions. Under a lower-level industry behaving as a Cournot oligopoly, however, regardless of the market design (TSO or MI), a greater fraction of generation comes from wind because producers withhold capacity. Their exercise of market power causes a welfare-maximizing TSO to subsidize wind to boost consumer surplus and a profit-maximizing MI to build more transmission lines in order to encourage more transmission flow. The somewhat counterintuitive result under Cournot leads to power flow from a wind producer to the node where a conventional producer was the sole incumbent. Finally, by implementing an RPS constraint requiring a given percentage of energy to be provided by renewable sources, we examine how the renewable-boosting outcome of the oligopoly may be attained even under perfect competition.

\section{Structure of the Paper}

In Section II, we present our modelling assumptions and the mathematical formulations for the capacity-expansion problems under various market designs. Next, in Section III, we implement numerical examples on a three-node network to gain insights about how market power and market design interact to drive investment outcomes. We conclude the paper in Section IV by summarizing our main points, discussing the limitations of our approach, and outlining directions for future research. The Appendix re-formulates Section II 's MPECs.

\section{PRoblem Formulation}

\section{A. Assumptions}

We assume that transmission capacity expansion can be made in discrete levels, $j \in \mathcal{J}_{\ell}$, for each line $\ell \in \mathcal{L}$ of the network. The susceptance of a line, $B_{\ell, j}$, and, thus, the power flow, $f_{\ell, j, t, s}$, depend on the chosen capacity level. Following [15], we use a DC load-flow approximation for network power flows, which is an acceptable convention in power systems economics as long as voltage angle differences are small, and assume that the realized power flow on line $\ell$ for capacity level $j$ in period $t$ and scenario $s$ is proportional to the susceptance and voltage angle difference, i.e., $f_{\ell, j, t, s}=u_{\ell, j} B_{\ell, j}\left(d_{n_{\ell, t, s}^{+}}-\right.$ $\left.d_{n_{\ell}^{-}, t, s}\right), \forall \ell, t, s, \forall j \in \mathcal{J}_{\ell}$. If capacity level $j$ is for line $\ell$, then $u_{\ell, j}=1$ with $f_{\ell, j, t, s}$ being positive or negative, depending on the direction of the flow. Furthermore, for $j^{\prime} \in \mathcal{J}_{\ell} \backslash\{j\}$, $u_{\ell, j^{\prime}}=0$ such that $f_{\ell, j^{\prime}, t, s}=0$ without any artificial constraints on the voltage angles. Thus, the realized flow on line $\ell$ can be computed as $\hat{f}_{\ell, t, s}=\sum_{j \in \mathcal{J}_{\ell}} f_{\ell, j, t, s}, \forall \ell, t, s$. As a result, the net imports at node $n$ are $\sum_{\ell \in \mathcal{L}_{n}^{-}} \hat{f}_{\ell, t, s}-\sum_{\ell \in \mathcal{L}_{n}^{+}} \hat{f}_{\ell, t, s}$.

Each producer is either conventional (using fossil fuel) or wind. Conventional producers, $i \in \mathcal{I}^{\mathrm{c}}$, have linear cost functions, while wind producers, $i \in \mathcal{I}^{\mathrm{w}}$, do not incur operational costs. In addition, conventional producers can decide how much to generate, whereas wind output is variable and non-dispatchable, i.e., determined by the availability factors $E_{n, t, s}$. Such 
variability in wind output may be due to differing wind potentials at various locations and uncertainty in future efficiency improvements of the technology. Other sources of variability, such as demand uncertainty, river inflows, and plant outages, likewise, drive investment decisions. However, whereas power companies may have years of experience in forecasting these sources, this may not be the case for the future availability of wind. Moreover, with the expected growth in renewables, wind power may account for a substantial share of variability in a future power system, and we take this as our main focus while acknowledging that it is not the only driver of investment. Meanwhile, since market rules like priority of wind production limit curtailment except in extreme situations, we assume wind to be non-dispatchable. Nevertheless, our model can easily be adapted to incorporate the dispatch of wind production. We account for variability in wind output by assuming a known and discrete distribution described by a number of scenarios capturing the wind availability factors $\left(E_{n, t, s}\right)$ and their probabilities $\left(P_{s}\right)$. Whereas conventional generation is typically controllable, other renewable energy sources, e.g., solar and run-of-river hydro, likewise exhibit variability in output and are non-dispatchable, and our approach could, therefore, also apply to them. We focus on wind, however, because its output tends to be more variable.

While any producer can install capacity at any node and sell electricity generated elsewhere by accessing transmission capacity, most power companies are well diversified and may own a portfolio of both conventional and wind plants. However, specialization also leads firms to concentrate on particular technologies, e.g., [26] report that the largest two firms in California have proportionately less conventional generation than the others as part of their portfolios. Thus, it is appropriate to think of each producer in our model as being a composite producer of a particular type (either predominantly conventional or predominantly wind). We assume that each node $n$ in our transmission grid has its own linear inverse demand, $p_{n, t, s}=A_{n}^{i n t}-A_{n}^{s l p} \sum_{i \in \mathcal{I}} q_{n, i, t, s}^{\text {sell }}$, in each period $t$ and scenario $s$, which depends on sales at the node by all producers in equilibrium. Depending on the market design, each producer is part of either a Cournot oligopoly or a perfectly competitive industry. The degree of market power is reflected by the conjectured price response, which is the first derivative of the inverse demand with respect to electricity sold by a given producer, i.e., $\partial p_{n, t, s} / \partial q_{n, i, t, s}^{\text {sell }}=-A_{n}^{s l p}\left(1+\sum_{i^{\prime} \in \mathcal{I} \backslash\{i\}} \Theta_{i^{\prime}, i, n}\right), \forall n, i, t, s$, where $\Theta_{i^{\prime}, i, n}=\partial q_{n, i^{\prime}, t, s}^{\text {sell }} / \partial q_{n, i, t, s}^{\text {sell }}$ for $i^{\prime} \in \mathcal{I} \backslash\{i\}$ and $\Theta_{i^{\prime}, i, n}=1$ otherwise. Hence, we model perfect competition and Cournot oligopoly when $\sum_{i^{\prime} \in \mathcal{I} \backslash\{i\}} \Theta_{i^{\prime}, i, n}$ equals -1 and 0 , respectively.

We formulate the transmission-expansion problem of the MI as a bi-level problem: transmission investment decisions are made at an upper level by the MI in anticipation of subsequent investment in and operation of wind and conventional generation capacity by the producers, transmission flow decisions of the MI, and market clearing, all captured by a number of lower-level problems. Effectively, we have a dominant MI investing in transmission capacity with wind and conventional producers as followers. We do not consider competing MIs for

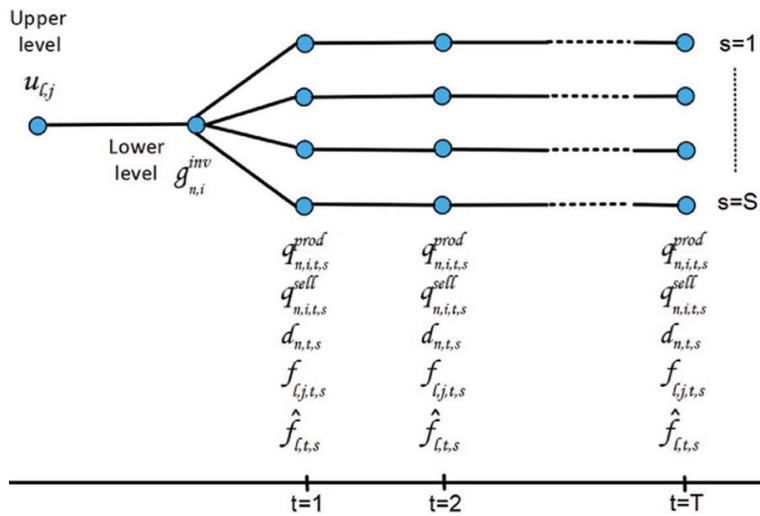

Fig. 1. Decision-making levels.

three reasons. First, only a few entities have the expertise to carry out transmission projects, e.g., the BritNed DC cable between the U.K. and The Netherlands was constructed in 2011 as a joint venture formed by a private holding company involving the National Grid and TenneT. Second, considering competing grid owners would typically involve a third decision-making level for procurement of transmission investment rights, which would make the problem a tri-level one [20] and, therefore, more computationally challenging. As we have mentioned, papers dealing with tri-level models for transmission planning typically do not solve them directly [19], [20]. By contrast, stylized tri-level models that can be solved explicitly abstract from attributes of the physical network [27], which is contrary to our objectives. Third, we would like to compare the MI and TSO market designs, and introducing a third level with procurement auctions would preclude such an analysis.

To approximate the impact on generation expansion and operational planning, the producers' problems are single level. This leads to an open-loop problem (MCP) for the producers rather than a more complicated closed-loop one (EPEC). While treating the producers' problems over two levels may impact our results based on the findings of [17], we use an open-loop representation of the producers' problems because the discrepancy between open- and closed-loop models occurs only for departures from a Cournot setting. Indeed, the imperfectly competitive nature of most electricity spot markets implies that results from the Cournot case are of greater significance, which is a central point of our paper and an issue that is relatively unaddressed in most hierarchical models of transmission investment. Although transmission and generation investment decisions are static, we allow for dynamic operational decisions and allocation of transmission capacity over time and scenarios. Thus, investment decisions are made in a first stage without anticipation of the wind output, whereas operational decisions are adapted to the realized scenario of availability factors, which leads to a two-stage stochastic program (Fig. 1). The stochastic bi-level problem can be re-formulated as an MPEC with equilibrium constraints obtained by deriving the optimality conditions for all lower-level problems. Since the lower level comprises convex optimization problems, their KKT conditions are sufficient for optimality.

As benchmarks, we consider market designs with either a welfare-maximizing central planner (CP) or a TSO. Since the CP 
controls all aspects of the energy market, it solves a single-level stochastic problem covering transmission and generation investment as well as generation dispatch and transmission flows. Like the MI, the TSO has a bi-level stochastic programming problem with all decisions made as per the MI market design. The only difference is that the TSO maximizes expected social welfare (SW) rather than expected profit from grid operations. Finally, in anticipation of forthcoming EU 2030 targets, we also run a numerical example with a stringent RPS target of $80 \%$. This is plausible because the $\mathrm{EU}$ will require a $40 \%$ reduction in $\mathrm{CO}_{2}$ emissions by 2030 relative to 1990 levels, which necessitates a deep decarbonization of the power sector specifically due to foreseen electrification of the transport sector. Hitting these targets will mean surmounting numerous technical challenges, but we are focused more on the implications of market design in such a transition assuming legally binding policy commitments.

\section{B. Merchant Investor}

1) MI's Upper-Level Problem: At the upper level, the MI decides on the transmission capacity level of a number of existing or potentially newly constructed transmission lines in order to maximize its expected profit given by the difference between grid congestion rents and investment costs:

$$
\begin{aligned}
\max _{\left\{u_{\ell, j}\right\}} & \sum_{s \in \mathcal{S}} P_{s} \sum_{t \in \mathcal{T}} \sum_{n \in \mathcal{N}} \tau_{n, t, s}\left(\sum_{\ell \in \mathcal{L}_{n}^{-}} \hat{f}_{\ell, t, s}-\sum_{\ell \in \mathcal{L}_{n}^{+}} \hat{f}_{\ell, t, s}\right) \\
& -\sum_{\ell \in \mathcal{L}} \sum_{j \in \mathcal{J}_{\ell}} C_{\ell, j}^{a r c} u_{\ell, j} \\
\text { s.t. } & u_{\ell, j} \in\{0,1\}, \forall \ell, \forall j \in \mathcal{J}_{\ell} \\
& \sum_{j \in \mathcal{J}_{\ell}} u_{\ell, j}=1, \forall \ell .
\end{aligned}
$$

Note that if $u_{\ell, j_{0}}=1$, then existing capacity remains and no new capacity is constructed. We model congestion pricing by assuming that all power flows through a hub node of the network without generation and consumption. We further assume that the MI charges a node-dependent congestion fee for transmitting power from this hub to each node [10]. The shadow price on market-clearing condition (33) sets the congestion fee. Upperlevel problem (1)-(3) is constrained by lower-level problems and equilibrium conditions.

2) MI's Lower-Level Problem: At the lower level, the MI determines flows on existing and newly constructed lines in order to maximize expected congestion rents:

$$
\begin{aligned}
\max _{\Gamma} & \sum_{s \in \mathcal{S}} P_{s} \sum_{t \in \mathcal{T}} \sum_{n \in \mathcal{N}} \tau_{n, t, s}\left(\sum_{\ell \in \mathcal{L}_{n}^{-}} \hat{f}_{\ell, t, s}-\sum_{\ell \in \mathcal{L}_{n}^{+}} \hat{f}_{\ell, t, s}\right) \\
\text { s.t. } & f_{\ell, j, t, s}=u_{\ell, j} B_{\ell, j}\left(d_{n_{\ell}^{+}, t, s}-d_{n_{\ell}^{-}, t, s}\right)\left(\psi_{\ell, j, t, s}\right) \\
& \forall \ell, t, s, \forall j \in \mathcal{J}_{\ell} \\
& f_{\ell, j, t, s} \leq K_{\ell, j}^{a r c}\left(\mu_{\ell, j, t, s}^{+}\right), \forall \ell, t, s, \forall j \in \mathcal{J}_{\ell} \\
& -f_{\ell, j, t, s} \leq K_{\ell, j}^{a r c}\left(\mu_{\ell, j, t, s}^{-}\right), \forall \ell, t, s, \forall j \in \mathcal{J}_{\ell} \\
& \hat{f}_{\ell, t, s}=\sum_{j \in \mathcal{J}_{\ell}} f_{\ell, j, t, s}\left(\kappa_{\ell, t, s}\right), \forall \ell, t, s \\
& S_{n} d_{n, t, s}=0\left(\xi_{n, t, s}\right), \forall n, t, s \\
& d_{n, t, s} \text { u.r.s., } \forall n, t, s ; f_{\ell, j, t, s} \text { u.r.s., } \forall \ell, t, s, \forall j \in \mathcal{J}_{\ell} \\
& \hat{f}_{\ell, t, s} \text { u.r.s., } \forall \ell, t, s
\end{aligned}
$$

where $\Gamma=\left\{\hat{f}_{\ell, t, s}, f_{\ell, j, t, s}, d_{n, t, s}\right\}$ and u.r.s. denotes variables of unrestricted sign. Constraint (5) defines the flow on each line for each capacity level as a function of the difference in voltage angles, transmission capacity choice (fixed at the upper level), and line susceptance [15]. The upper and lower limits on transmission flows for each capacity level of each line are set by (6) and (7), respectively, while (8) indicates the realized flow on each line. Restrictions (9) set the slack node for calculating voltage angles of the network. Moreover, the corresponding dual variables are in brackets.

The KKT conditions for the MI's lower-level problem are

$$
\begin{aligned}
& -P_{s}\left(\tau_{n_{\ell}^{-}, t, s}-\tau_{n_{\ell}^{+}, t, s}\right)+\kappa_{\ell, t, s}=0 \text { with } \hat{f}_{\ell, t, s} \text { u.r.s., } \\
& \forall \ell, t, s \\
& \psi_{\ell, j, t, s}+\mu_{\ell, j, t, s}^{+}-\mu_{\ell, j, t, s}^{-}-\kappa_{\ell, t, s}=0 \text { with } f_{\ell, j, t, s} \text { u.r.s., } \\
& \forall \ell, t, s, \forall j \in \mathcal{J}_{\ell} \\
& \quad-\sum_{\ell \in \mathcal{L}_{n}^{+}} \sum_{j \in \mathcal{J}_{\ell}} u_{\ell, j} B_{\ell, j} \psi_{\ell, j, t, s}+\sum_{\ell \in \mathcal{L}_{n}^{-}} \sum_{j \in \mathcal{J}_{\ell}} u_{\ell, j} B_{\ell, j} \psi_{\ell, j, t, s} \\
& +S_{n} \xi_{n, t, s}=0 \text { with } d_{n, t, s} \text { u.r.s., } \forall n, t, s \\
& f_{\ell, j, t, s}-u_{\ell, j} B_{\ell, j}\left(d_{n_{\ell}^{+}, t, s}-d_{n_{\ell}^{-}, t, s}\right)=0 \\
& \text { with } \psi_{\ell, j, t, s} \text { u.r.s., } \forall \ell, t, s, \forall j \in \mathcal{J}_{\ell} \\
& 0 \leq K_{\ell, j}^{a r c}-f_{\ell, j, t, s} \perp \mu_{\ell, j, t, s}^{+} \geq 0, \forall \ell, t, s, \forall j \in \mathcal{J}_{\ell} \\
& 0 \leq K_{\ell, j}^{a r c}+f_{\ell, j, t, s} \perp \mu_{\ell, j, t, s}^{-} \geq 0, \forall \ell, t, s, \forall j \in \mathcal{J}_{\ell} \\
& \hat{f}_{\ell, t, s}-\sum_{j \in \mathcal{J}_{\ell}} f_{\ell, j, t, s}=0 \text { with } \kappa_{\ell, t, s} \text { u.r.s., } \forall \ell, t, s \\
& S_{n} d_{n, t, s}=0 \text { with } \xi_{n, t, s} \text { u.r.s., } \forall n, t, s .
\end{aligned}
$$

3) Producers' Lower-Level Problems: Each conventional producer $i \in \mathcal{I}^{\mathrm{c}}$ decides on investment and operation of generating units by maximizing expected profit. This is revenue minus congestion rent, operating cost, compliance cost with the RPS stemming from renewable energy certificates (RECs), and investment cost:

$$
\begin{aligned}
\max _{\Gamma_{i}} & \sum_{s \in \mathcal{S}} P_{s} \sum_{t \in \mathcal{T}} \sum_{n \in \mathcal{N}}\left(\left(A_{n}^{\text {int }}-A_{n}^{s l p} \sum_{i^{\prime} \in \mathcal{I}} q_{n, i^{\prime}, t, s}^{\text {sell }}\right.\right. \\
& \left.\left.-\tau_{n, t, s}\right) q_{n, i, t, s}^{\text {sell }}-\left(C_{n, i}^{\text {prod }}-\tau_{n, t, s}+R v_{t, s}\right) q_{n, i, t, s}^{\text {prod }}\right) \\
& -\sum_{n \in \mathcal{N}} C_{n, i}^{\text {inv }} g_{n, i}^{\text {inv }} \\
\text { s.t. } & q_{n, i, t, s}^{\text {prod }} \leq K_{n, i}^{\text {prod }}+g_{n, i}^{\text {inv }}\left(\lambda_{n, i, t, s}^{\mathrm{c}}\right), \forall n, t, s \\
& \sum_{n \in \mathcal{N}} q_{n, i, t, s}^{\text {prod }}-\sum_{n \in \mathcal{N}} q_{n, i, t, s}^{\text {sell }}=0\left(\theta_{i, t, s}\right), \forall t, s \\
& q_{n, i, t, s}^{\text {prod }} \geq 0, q_{n, i, t, s}^{\text {sell }} \geq 0, \forall n, t, s, g_{n, i}^{\text {inv }} \geq 0, \forall n .
\end{aligned}
$$

Here, $\Gamma_{i}=\left\{q_{n, i, t, s}^{\text {prod }}, q_{n, i, t, s}^{\text {sell }}, g_{n, i}^{\text {inv }}\right\}$. Congestion pricing implies that a producer receives/makes a payment to send power from the generation node/hub node to the hub node/sales node as in [10]. These payments are again shadow prices from the market-clearing condition (33). The problem is subject to capacity constraints on production (20) and energy balance between total production and sales (21). Following [25], we account for the REC payment via the exogenous RPS fraction, $R$, and the shadow price on the RPS constraint (34). 
The KKT conditions for this problem are

$$
\begin{aligned}
& 0 \leq-P_{s}\left(A_{n}^{i n t}-A_{n}^{s l p}\left(\left(1+\sum_{i^{\prime} \in \mathcal{I} \backslash\{i\}} \Theta_{i, i^{\prime}, n}\right) q_{n, i, t, s}^{\text {sell }}\right.\right. \\
& \left.\left.+\sum_{i^{\prime} \in \mathcal{I}} q_{n, i^{\prime}, t, s}^{\text {sell }}\right)-\tau_{n, t, s}\right)+\theta_{i, t, s} \perp q_{n, i, t, s}^{\text {sell }} \geq 0, \forall n, t, s(23) \\
& 0 \leq P_{s}\left(C_{n, i}^{\text {prod }}-\tau_{n, t, s}+R v_{t, s}\right)+\lambda_{n, i, t, s}^{\mathrm{c}}-\theta_{i, t, s} \\
& \quad \perp q_{n, i, t, s}^{\text {prod }} \geq 0, \forall n, t, s \\
& 0 \leq C_{n, i}^{\text {rnv }}-\sum_{s \in \mathcal{S}} \sum_{t \in \mathcal{T}} \lambda_{n, i, t, s}^{\mathrm{c}} \perp g_{n, i}^{i n v} \geq 0, \forall n \\
& 0 \leq K_{n, i}^{\text {prod }}+g_{n, i}^{i n v}-q_{n, i, t, s}^{\text {prod }} \perp \lambda_{n, i, t, s}^{\mathrm{c}} \geq 0, \forall n, t, s \\
& \sum_{n \in \mathcal{N}} q_{n, i, t, s}^{\text {prod }}-\sum_{n \in \mathcal{N}} q_{n, i, t, s}^{\text {sell }}=0 \text { with } \theta_{i, t, s} \text { u.r.s. }, \forall t, s .(27)
\end{aligned}
$$

Each wind power producer $i \in \mathcal{I}^{\mathrm{w}}$ faces a similar problem:

$$
\begin{aligned}
\max _{\Gamma_{i}} & \sum_{s \in \mathcal{S}} P_{s} \sum_{t \in \mathcal{T}} \sum_{n \in \mathcal{N}}\left(\left(A_{n}^{\text {int }}-A_{n}^{\text {slp }} \sum_{i \in \mathcal{I}} q_{n, i, t, s}^{\text {sell }}\right.\right. \\
& \left.\left.-\tau_{n, t, s}\right) q_{n, i, t, s}^{\text {sell }}+\left(\tau_{n, t, s}+(1-R) v_{t, s}\right) q_{n, i, t, s}^{\text {prod }}\right) \\
& -\sum_{n \in \mathcal{N}} C_{n, i}^{\text {inv }} g_{n, i}^{\text {inv }} \\
\text { s.t. } & q_{n, i, t, s}^{\text {prod }}=E_{n, t, s}\left(K_{n, i}^{\text {prod }}+g_{n, i}^{\text {inv }}\right)\left(\lambda_{n, i, t, s}^{\mathrm{w}}\right) \\
& \forall n, t, s
\end{aligned}
$$$$
\text { (21), (22). }
$$

Note that in the objective function production costs of wind power production are assumed to be negligible and REC payments are replaced by earnings from RECs. Furthermore, the constraint (20) is replaced by (29) to reflect the non-dispatchable nature of wind.

The corresponding KKT conditions are

$(23),(27)$

$$
\begin{aligned}
0 \leq & -P_{s}\left(\tau_{n, t, s}+(1-R) v_{t, s}\right)+\lambda_{n, i, t, s}^{\mathrm{w}}-\theta_{i, t, s} \\
& \perp q_{n, i, t, s}^{\text {prod }} \geq 0, \forall n, t, s \\
0 \leq & C_{n, i}^{i n v}-\sum_{s \in \mathcal{S}} \sum_{t \in \mathcal{T}} \lambda_{n, i, t, s}^{\mathrm{w}} E_{n, t, s} \perp g_{n, i}^{i n v} \geq 0, \forall n \\
& q_{n, i, t, s}^{\text {prod }}-E_{n, t, s}\left(K_{n, i}^{\text {prod }}+g_{n, i}^{i n v}\right)=0 \text { with } \lambda_{n, i, t, s}^{\mathrm{w}} \text { u.r.s. } \\
& \forall n, t, s .
\end{aligned}
$$

4) Equilibrium Conditions: Finally, the market-clearing and the RPS constraints are

$$
\begin{aligned}
& \sum_{i \in \mathcal{I}} q_{n, i, t, s}^{\text {sell }}-\sum_{i \in \mathcal{I}} q_{n, i, t, s}^{\text {prod }}+\sum_{\ell \in \mathcal{L}_{n}^{+}} \hat{f}_{\ell, t, s}-\sum_{\ell \in \mathcal{L}_{n}^{-}} \hat{f}_{\ell, t, s}=0, \\
& \text { with } \tau_{n, t, s} \text { u.r.s., } \forall n, t, s \\
& 0 \leq \sum_{n \in \mathcal{N}} \sum_{i \in \mathcal{I}^{\mathrm{w}}} q_{n, i, t, s}^{\text {prod }}-R \sum_{n \in \mathcal{N}} \sum_{i \in \mathcal{I}} q_{n, i, t, s}^{\text {prod }} \perp v_{t, s} \geq 0 \\
& \forall t, s .
\end{aligned}
$$

\section{5) MI's MPEC Formulation: The MI's MPEC is}

$\max _{\left\{u_{\ell, j}\right\} \cup \Gamma \cup \Gamma_{i} \cup-10000 f_{i l^{M I}}}(1)$

s.t. (2)-(3); (11)-(18); (23), (27) $\forall i \in \mathcal{I}$;

$(24)-(26) \forall i \in \mathcal{I}^{\mathrm{c}} ;(30)-(32) \forall i \in \mathcal{I}^{\mathrm{w}}$;

(33) $-(34)$

where $-10000 f i l^{M I}=\left\{\mu_{\ell, j, t, s}^{+}, \mu_{\ell, j, t, s}^{-}, \psi_{\ell, j, t, s}, \kappa_{\ell, t, s}, \xi_{n, t, s}\right.$, $\left.\lambda_{n, i, t, s}^{\mathrm{c}}, \lambda_{n, i, t, s}^{\mathrm{w}}, \theta_{i, t, s}, \tau_{n, t, s}, v_{t, s}\right\}$ are lower-level dual variables.

\section{Transmission System Operator}

At the upper level, the TSO decides on transmission capacity such as to maximize expected social welfare:

$$
\begin{aligned}
\max _{\left\{u_{\ell, j}\right\}} & \sum_{s \in \mathcal{S}} P_{s} \sum_{t \in \mathcal{T}}\left(\sum _ { n \in \mathcal { N } } \left(A_{n}^{i n t} \sum_{i \in \mathcal{I}} q_{n, i, t, s}^{\text {sell }}\right.\right. \\
& \left.\left.-\frac{1}{2} A_{n}^{s l p}\left(\sum_{i \in \mathcal{I}} q_{n, i, t, s}^{s e l l}\right)^{2}\right)-\sum_{i \in \mathcal{I}^{\mathrm{c}}} \sum_{n \in \mathcal{N}} C_{n, i}^{\text {prod }} q_{n, i, t, s}^{\text {prod }}\right) \\
& -\sum_{i \in \mathcal{I}} \sum_{n \in \mathcal{N}} C_{n, i}^{i n v} g_{n, i}^{i n v}-\sum_{\ell \in \mathcal{L}} \sum_{j \in \mathcal{J}_{\ell}} C_{\ell, j}^{a r c} u_{\ell, j}
\end{aligned}
$$$$
\text { s.t. }(2)-(3) \text {. }
$$

At the lower level, the TSO enforces network feasibility constraints, (5) -(10), in which $u_{\ell, j}$ is fixed at the upper level. Thus, the TSO's MPEC is

$\max _{\left\{u_{\ell, j}\right\} \cup \Gamma \cup \Gamma_{i} \cup-10000 f i l^{T S O}}$

s.t. (2)-(3); (5)-(10); (23), (27) $\forall i \in \mathcal{I}$;

$(24)-(26) \forall i \in \mathcal{I}^{\mathrm{c}} ;(30)-(32) \forall i \in \mathcal{I}^{\mathrm{w}}$;

$(33)-(34)$

where $-10000 \mathrm{fil}^{T S O}=\left\{\lambda_{n, i, t, s}^{\mathrm{c}}, \lambda_{n, i, t, s}^{\mathrm{w}}, \theta_{i, t, s}, \tau_{n, t, s}, v_{t, s}\right\}$.

\section{Central Planner}

The CP's stochastic mixed-integer non-linear programming (MINLP) problem is

$$
\begin{aligned}
& \max _{\left\{u_{\ell, j}\right\} \cup \Gamma \cup \Gamma_{i}}(35) \\
& \text { s.t. }(2)-(3) ;(5)-(10) ;(20) \forall i \in \mathcal{I}^{\mathrm{c}} ; \\
&(21)-(22) \forall i \in \mathcal{I} ;(29) \forall i \in \mathcal{I}^{\mathrm{w}} ;(33) ; \\
& \sum_{n \in \mathcal{N}} \sum_{i \in \mathcal{I}^{\mathrm{w}}} q_{n, i, t, s}^{\text {prod }} \geq R \sum_{n \in \mathcal{N}} \sum_{i \in \mathcal{I}} q_{n, i, t, s}^{\text {prod }} \\
& \forall n, t, s .
\end{aligned}
$$

\section{NUMERICAL EXAMPLES}

\section{A. Data}

We implement the three market designs on a three-node network with two operating hours and scenarios (Fig. 2). The arrows indicate forward directions for the flows, i.e., the corresponding decision variable will have a positive (negative) sign if the realized flow is in the indicated (opposite) direction. All 


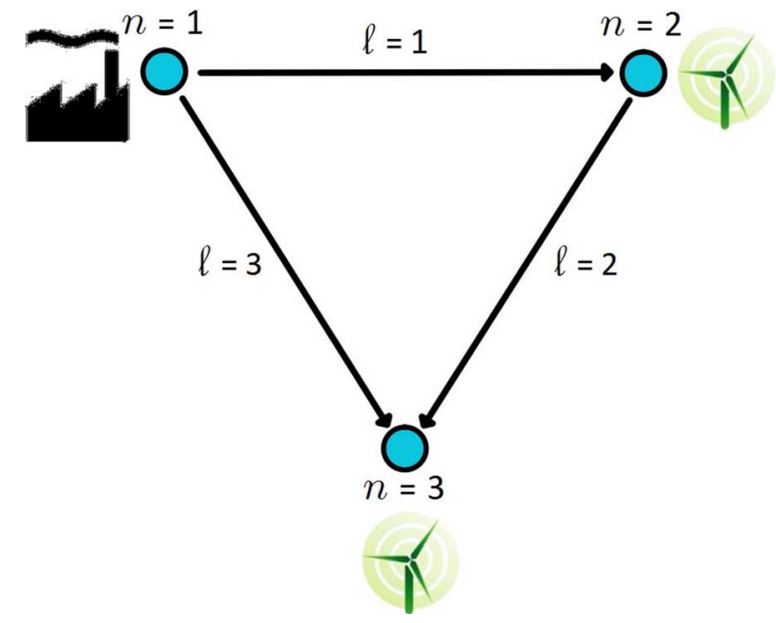

Fig. 2. Transmission network.

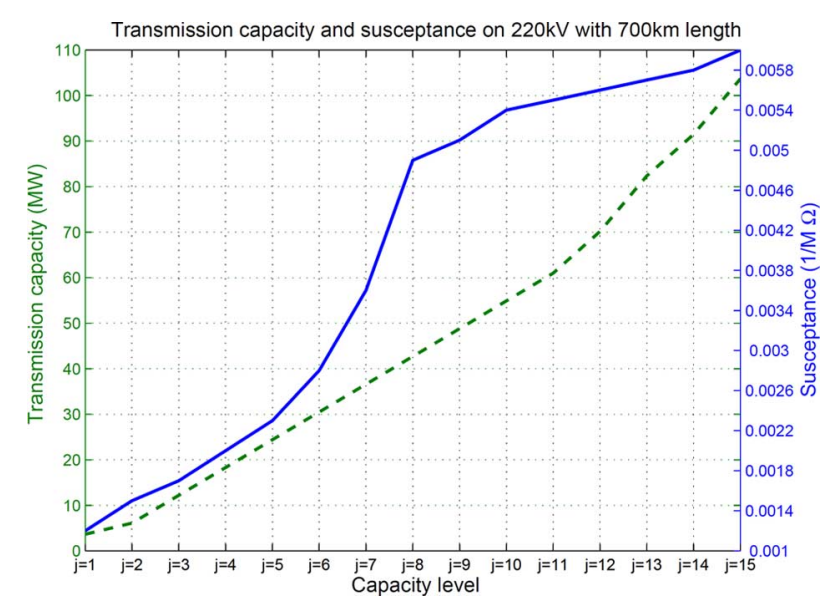

Fig. 3. Transmission line parameters.

nodes are initially disconnected, but transmission lines with attributes given in Fig. 3 may be built. Here, the transmission capacity (susceptance) is indicated by the broken (solid) series measured on the left (right) axis. Specifically, we consider fifteen discrete capacity levels with corresponding susceptances calculated according to [28]. Although in our example, the network is initially disconnected, because we discretize the transmission capacity levels, we could easily implement an instance with positive initial line capacities. Since we model representative hours, the transmission investment costs for lines of length $700 \mathrm{~km}$ are amortized on an hourly basis assuming a construction cost of $\$ 1080 /(\mathrm{MW}-\mathrm{km})$, which is in line with [15]. These costs range from $€ 11.9 / \mathrm{MW}$ to $€ 337 / \mathrm{MW}$ for 220 -kV lines corresponding to capacities $3.7 \mathrm{MW}$ to $103.7 \mathrm{MW}$, respectively. For generation, we use the US Energy Information Administration's 2014 Annual Energy Outlook to calculate operating costs and amortized capacity costs of conventional $(\$ 2930 / \mathrm{kW}$ capital cost and $40 \%$ efficiency for coal) and wind plants (capital costs of $\$ 2210 / \mathrm{kW}$ and $\$ 6230 / \mathrm{kW}$ for onshore and offshore turbines, respectively). All amortization assumes a lifetime of 20 years and an interest rate of $3 \%$ per annum. Finally, in Table I, we assume that the demand centre is at node 1 (with an existing conventional plant), but wind resources are based at thinly populated locations (nodes 2 and 3 ).
TABLE I

DEMAND AND PRODUCTION PARAMETERS

\begin{tabular}{|l||lll|}
\hline \hline Parameter & $n=1$ & $n=2$ & $n=3$ \\
& $i=1$ & $i=2$ & $i=3$ \\
\hline$A_{n}^{\text {int }}$ & 110 & 70 & 60 \\
$A_{n}^{\text {slp }}$ & 1 & 1 & 1 \\
$E_{n, s, 1}$ & 0 & 0.30 & 0.45 \\
$E_{n, 1,2}$ & 0 & 0.33 & 0.50 \\
$E_{n, 2,2}$ & 0 & 0.27 & 0.41 \\
$C_{n, i}^{\text {inv }}$ & 12.58 & 9.47 & 26.71 \\
$C_{n, i}^{\text {prod }}$ & 21 & 0 & 0 \\
$K_{n, i}^{\text {prod }}$ & 15 & 0 & 0 \\
\hline
\end{tabular}

\section{B. Computational Issues}

The problems are implemented in GAMS running on a Windows workstation with a 3.30-GHz Intel i7 core processor and $16 \mathrm{~GB}$ of RAM. While the CP's MINLP and the TSO's MPEC are re-formulated as mixed-integer quadratic programs (MIQPs), the MI's MPEC is re-formulated as an MILP (see the Appendix). All problems are solved via GUROBI. Computational times with two periods and two scenarios range from less than one second (CP and all TSO instances) to $855 \mathrm{~s}$ (MI with $\mathrm{PC}$ ) and $7735 \mathrm{~s}$ (MI with $\mathrm{CO}$ ). In the latter instance, there are 3481 equations, 1520 continuous variables, and 484 discrete variables. The relative optimality gap is set to $5 \%$.

\section{Example 1: Base Case Without RPS}

As a benchmark, we find that the CP simply matches the most efficient resource with the most valuable demand nodes (Table II). The conventional producer at node 1 serves all of the local demand, while transmission lines are constructed from nodes 2 to 3 with an effectively zero expected profit, $\mathbb{E}\left[\overline{\Pi_{n, i}}\right]$, for producers if the subsidy on congestion rent from the $\mathrm{CP}$ and the legacy capacity for the conventional producer are ignored. Moreover, the expected profit from grid operations, $\mathbb{E}\left[\overline{\bar{\Pi}^{\text {arc }}}\right]$, is negative as congestion rents are internalized in a centrally planned economy. Meanwhile, the TSO's result under perfect competition is similar to that of the $\mathrm{CP}$ aside from the levying of congestion rents, which drives the producers' expected profits to zero (with the exception of the conventional producer). This is in contrast to [19] because of the difference in formulation: we have sales and dispatch decisions made by producers, whereas [19] assumes that only dispatch is made by producers with sales and flow decisions performed by a welfare-maximizing TSO. The MI under perfect competition delivers a lower social welfare because of its incentive to maximize its own expected profit, which is attained by reducing line capacities to boost congestion rents. Consequently, the producers adopt less generation capacity, and the expected nodal prices and differences in expected congestion rents are higher. For example, under the MI, it costs a perfectly competitive producer at node 2 nearly $€ 16 / \mathrm{MW}$ to send power to node 3 as opposed to about $€ 4 / \mathrm{MW}$ with a TSO. Hence, the MI delivers lower social welfare and less renewable generation than the TSO. 
TABLE II

RESULTS FOR EXAMPLE 1

\begin{tabular}{|c|c|c|c|c|c|}
\hline & \multicolumn{2}{|c|}{ Cournot Oligopoly } & \multicolumn{2}{|c|}{ Perfect Competition } & \multirow[t]{2}{*}{$\mathrm{CP}$} \\
\hline & MI & TSO & MI & TSO & \\
\hline $\mathbb{E}[\overline{S W}]$ & 3549.50 & 3860.19 & 4073.66 & 4154.39 & 4154. \\
\hline $\mathbb{E}[\overline{\mathrm{RG}}](\%)$ & 46.80 & 87.83 & 39.65 & 44.83 & 44.8 \\
\hline $\mathbb{E}\left[\overline{\Pi^{a r c}}\right]$ & 63.72 & -4351.73 & 155.27 & 20.97 & 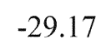 \\
\hline$\sum_{i \in \mathcal{T}_{1}} u_{1, j} K_{1, j}^{a r c}$ & 3.70 & 61.00 & 0.00 & 0.00 & 0.00 \\
\hline$\sum_{i \in \mathcal{T}_{0}}^{j \in \mathcal{J}_{1}} u_{2, j} K_{2, j}^{a r c}$ & 6.10 & 30.50 & 12.20 & 24.40 & 24. \\
\hline$\sum_{j \in \mathcal{J}_{3}} u_{3, j} K_{3, j}^{a r c}$ & 0.00 & 0.00 & 0.00 & 0.00 & 0.00 \\
\hline $\mathbb{E}[\overline{\text { flow }} 1]$ & -3.67 & -58.45 & 0.00 & 0.00 & 0.00 \\
\hline $\mathbb{E}\left[\right.$ flow $\left._{2}\right]$ & 6.10 & 29.52 & 12.20 & 24.05 & 24.05 \\
\hline $\mathbb{E}\left[\right.$ flow $\left._{3}\right]$ & 0.00 & 0.00 & 0.00 & 0.00 & 0.00 \\
\hline $\mathbb{E}\left[\overline{p_{1}}\right]$ & 58.41 & 36.81 & 8 & 33.58 & 33.58 \\
\hline $\mathbb{E}\left[\overline{p_{2}}\right]$ & 43.31 & 51.06 & 31.8 & 31.8 & 31.84 \\
\hline $\mathbb{E}\left[\bar{p}_{3}\right]$ & 48.17 & 30.48 & 47.80 & 35.95 & 35.95 \\
\hline $\mathbb{E}\left[\overline{\tau_{1}}\right]$ & -1.76 & 22.31 & 0.47 & -0.16 & - \\
\hline $\mathbb{E}\left[\overline{\tau_{2}}\right]$ & 0.07 & 76.07 & 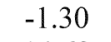 & -1.90 & - \\
\hline $\mathbb{E}\left[\bar{\tau}_{3}\right]$ & -3.18 & 44.90 & 14.69 & 2.21 & - \\
\hline $\mathbb{E}\left[\overline{\overline{\Pi_{1,1}}}\right]$ & 1100.45 & 233.60 & 188.70 & 188.70 & 182.6 \\
\hline $\mathbb{E}\left[\overline{\Pi_{2,2}}\right]$ & 592.09 & 4672.03 & 0.00 & 0.0 & 56.15 \\
\hline $\mathbb{E}\left[\overline{\Pi_{3,3}}\right]$ & 33.05 & 0.00 & 0.00 & 0.00 & 0.00 \\
\hline$g_{1.1}^{i n v}$ & 32.91 & 0.00 & 61.42 & 61.42 & 61.42 \\
\hline$g_{2,2}^{i n v}$ & 121.56 & 356.34 & 167.94 & 207.35 & 207.35 \\
\hline$g_{3.3}^{i n v}$ & 12.74 & 0.00 & 0.00 & 0.00 & 0.00 \\
\hline
\end{tabular}

Under a Cournot oligopoly, producers have the incentive to withhold generation capacity in order to boost expected profits. Anticipating this strategic behavior, the TSO supports expected SW by effectively subsidizing wind generation by investing heavily in line 1 . This creates an opening for the wind producer at node 2 to benefit at the expense of the conventional producer at node 1. Consequently, the generation investment and operations observed under perfect competition are altered as a result of the exercise of market power by the producers and the countervailing decisions of the TSO at the upper level. For an MI, the strategic withholding by producers at the lower level is likewise undesirable because it cuts into transmission flows. Recognizing this, the MI mitigates losses to its profit by encouraging transmission. Thus, for different reasons than the TSO, the MI also invests in more distinct lines under Cournot oligopoly than under perfect competition. However, total transmission capacity drops under the Cournot setting because of the producers' propensity to withhold generation and the MI's reluctance to subsidize wind to increase social welfare. Finally, although the MI's actions result in wind investment at node 3, expected renewable generation, $\mathbb{E}[\overline{\mathrm{RG}}]$, is greatest under the TSO.

\section{Example 2: Renewable Portfolio Standards}

Given that the EU's 2030 objective is to decrease $\mathrm{CO}_{2}$ emissions by $40 \%$ relative to 1990 levels, i.e., implying a deep decarbonization of the power sector, we run our model with an RPS target of $80 \%$ (Table III). We find that the effective subsidy for wind (and tax on conventional generation) enables the high penetration of renewables seen under Cournot oligopoly in Table II to be achieved here even under perfect competition.
TABLE III

RESULTS FOR EXAMPLE 2

\begin{tabular}{|c|c|c|c|c|c|}
\hline & \multicolumn{2}{|c|}{ Cournot Oligopoly } & \multicolumn{2}{|c|}{ Perfect Competition } & \multirow[t]{2}{*}{$\mathrm{CP}$} \\
\hline & MI & TSO & MI & TSO & \\
\hline $\mathbb{E}[\overline{S W}]$ & 2705.35 & 3860.18 & 3084.858 & 4073.84 & 4073.84 \\
\hline $\mathbb{E}[\overline{\mathrm{RG}}](\%)$ & 80.10 & 87.80 & 80.10 & 81.57 & 81.57 \\
\hline $\mathbb{E}[\bar{v}]$ & 48.85 & 0.00 & 49.07 & 4.09 & - \\
\hline $\mathbb{E}\left[\overline{\Pi^{a r c}}\right]$ & 576.93 & -4351.73 & 1010.291 & 158.68 & -39.61 \\
\hline$\sum_{j \in \mathcal{T}} u_{1, j} K_{1, j}^{a r c}$ & 12.20 & 61.0 & 18.30 & 48.80 & 48.80 \\
\hline$\sum_{j \in \mathcal{J}_{2}}^{j \in \mathcal{J}_{1}} u_{2, j} K_{2, j}^{a r c}$ & 12.20 & 30.5 & 6.10 & 24.40 & 24.40 \\
\hline$\sum_{j \in \mathcal{J}_{3}} u_{3, j} K_{3, j}^{a r c}$ & 3.70 & 0.00 & 0.00 & 0.00 & 0.00 \\
\hline $\mathbb{E}\left[\overline{f l o w_{1}}\right]$ & -12.20 & -58.44 & -18.30 & -48.25 & -48.25 \\
\hline $\mathbb{E}\left[\overline{f l o w_{2}}\right]$ & 8.08 & 29.524 & 6.10 & 23.68 & 23.68 \\
\hline $\mathbb{E}\left[\overline{\text { llow }_{3}}\right]$ & -3.70 & 0.00 & 0.00 & 0.00 & 0.00 \\
\hline $\mathbb{E}\left[\overline{p_{1}}\right]$ & 79.80 & 36.80 & 72.83 & 36.85 & 36.85 \\
\hline $\mathbb{E}\left[\overline{p_{2}}\right]$ & 40.94 & 51.06 & 22.44 & 31.26 & 31.26 \\
\hline $\mathbb{E}\left[\overline{p_{3}}\right]$ & 47.48 & 30.47 & 49.89 & 36.33 & 36.33 \\
\hline $\mathbb{E}\left[\overline{\tau_{1}}\right]$ & -10.48 & 11.94 & -9.698 & 2.80 & - \\
\hline $\mathbb{E}\left[\overline{\tau_{2}}\right]$ & 3.50 & 11.65 & 0.076 & 0.00 & - \\
\hline $\mathbb{E}\left[\overline{\tau_{3}}\right]$ & -0.32 & 13.44 & 0.00 & 2.50 & - \\
\hline $\mathbb{E}\left[\overline{\Pi_{1,1}}\right]$ & 286.29 & 233.60 & 188.70 & 188.70 & 243.42 \\
\hline $\mathbb{E}\left[\overline{\Pi_{2,2}}\right]$ & 844.81 & 4672.02 & 0.00 & 0.00 & 143.57 \\
\hline $\mathbb{E}\left[\overline{\Pi_{3,3}}\right]$ & 33.73 & 0.00 & 0.00 & 0.00 & 0.00 \\
\hline$g_{1,1}^{i n v}$ & 0.51 & 0.00 & 5.38 & 9.90 & 9.90 \\
\hline$g_{2,2}^{i n v}$ & 164.49 & 356.34 & 239.84 & 368.89 & 368.89 \\
\hline$g_{3,3}^{i n v}$ & 18.09 & 0.00 & 8.897 & 0.00 & 0.00 \\
\hline
\end{tabular}

In effect, RPS mimics the high renewable penetration with alteration of generation patterns under oligopoly. A numerical example with a $60 \%$ RPS target has similar qualitative results, i.e., a higher penetration of wind under perfect competition.

\section{E. Larger Problem Instances}

In order to investigate the robustness of our insights, we also implement two additional examples. For the three-node network with four scenarios and eight time periods, computational times for the CP and TSO market designs are again less than one second. However, those for the MI market designs balloon to $9 \mathrm{~h}$ (with PC) and $35 \mathrm{~h}$ (with CO). Nevertheless, the qualitative insights are similar to those in Example 1. For a six-node network with eight candidate transmission lines, computational times for the CP and TSO market designs are about four minutes, and the results are as for the three-node network, i.e., transmission lines are built to transfer the wind power to consumption centres. However, the MI market designs become more challenging to solve without recourse to decomposition, which is an area for future work.

\section{Discussion AND CONCLUSIONS}

Deregulation of the power sector has created various market designs to balance competing economic and social objectives. Here, we take a complementarity approach to compare MI and TSO market designs in analyzing a transition to a more sustainable electricity industry. By re-formulating the bi-level problems as MPECs and then as MILPs or MIQPs, we implement these market designs for a three-node example and along with an RPS requirement. We demonstrate how market design and market power interact to result in seemingly counterintuitive 
outcomes. In particular, we note that under the CP and TSO (with perfect competition) market designs, the results are similar. Intuitively, the conventional producer satisfies all of the local demand, while a transmission line linking nodes with wind producers is constructed. This results in generation expansion by the conventional producer and the cheaper (on-shore) wind producer, and power flows towards the location of the more expensive (off-shore) wind producer. The MI market design under perfect competition is qualitatively similar in terms of transmission investment, generation expansion, and power flows. However, since the MI is concerned about maximizing its own expected profit only, it strategically invests in less transmission capacity to increase congestion rents, thereby earning positive expected profit. The expected generation from renewables is similar to that under the CP and TSO (with perfect competition) market designs.

Allowing for market power at the lower level leads to less generation investment as producers seek to drive up the market-clearing price. Under the TSO market design with a Cournot oligopoly, the conventional producer's act of withholding investment, indeed, increases its own expected profit and average prices across the network. However, this withholding could have a deleterious effect on social welfare, which the TSO seeks to mitigate by effectively subsidizing more transmission investment along lines involving the on-shore wind producer. Consequently, this countervailing action by the TSO creates an opening for the on-shore wind producer to expand generation capacity in order to offset some of the effects of the market power exercised by the conventional producer. Somewhat counterintuitively, expected generation from renewables increases significantly with a Cournot oligopoly vis-à-vis perfect competition, and the on-shore wind producer actually exports power to the conventional producer's node.

This rather surprising result is also encountered in the MI market design with a Cournot oligopoly but for different reasons. As with the TSO, the lower level now has producers with the incentives to withhold generation capacity in order to increase expected profits and prices. However, such behavior will conflict with the MI's objective to maximize expected profit, which consists of the product of congestion rents and power flows. Since the latter are adversely affected by the producers' withholding, the MI takes countermeasures to prevent power flows from dropping too much. Thus, it also invests in lines connecting the on-shore wind producer with the rest of the network, albeit by much less than the TSO. This enables power generation by not only the on-shore wind producer but also the more expensive off-shore wind producer. With an RPS constraint, the enhanced role for wind producers supported by complementary transmission investment is observed even when the lower level does not have producers behaving à la Cournot. Hence, the desirable policy target of significant renewable penetration may be attained (even in the presence of conflicting game-theoretic incentives) without the need to tolerate market power.

Although our work reflects salient features of the power sector such as strategic behavior, loop flows, and variable, non-dispatchable wind output under different market designs, it may be enhanced further. Possible pathways for extension are implementation of the models on more realistic test networks, a larger number of scenarios for wind output, and a bi-level representation of producer behavior, which will necessitate recourse to decomposition algorithms.

\section{APPENDIX \\ PROBLEM RE-FORMULATIONS}

We re-formulate the MPECs in Section II as mixed-integer linear programs (MILPs) or mixed-integer quadratic programs (MIQPs). Starting with the MI's objective function, we convexify its non-convex terms by applying strong duality from linear programming (LP) to its lower-level problem:

$$
\begin{aligned}
\sum_{s \in \mathcal{S}} P_{s} \sum_{t \in \mathcal{T}} \sum_{n \in \mathcal{N}} \tau_{n, t, s}\left(\sum_{\ell \in \mathcal{L}_{n}^{-}} \hat{f}_{\ell, t, s}-\sum_{\ell \in \mathcal{L}_{n}^{+}} \hat{f}_{\ell, t, s}\right) \\
=\sum_{s \in \mathcal{S}} \sum_{t \in \mathcal{T}} \sum_{\ell \in \mathcal{L}} \sum_{j \in \mathcal{J}_{\ell}} K_{\ell, j}^{a r c}\left(\mu_{\ell, j, t, s}^{+}+\mu_{\ell, j, t, s}^{-}\right) .
\end{aligned}
$$

Consequently, the non-convex part of the MI's objective function can be replaced by a linear expression to yield the following objective function:

$$
\begin{aligned}
& \sum_{s \in \mathcal{S}} \sum_{t \in \mathcal{T}} \sum_{\ell \in \mathcal{L}} \sum_{j \in \mathcal{J}_{\ell}} K_{\ell, j}^{a r c}\left(\mu_{\ell, j, t, s}^{+}+\mu_{\ell, j, t, s}^{-}\right) \\
&-\sum_{\ell \in \mathcal{L}} \sum_{j \in \mathcal{J}_{\ell}} C_{\ell, j}^{a r c} u_{\ell, j} .
\end{aligned}
$$

For this to hold, the primal and dual solutions, $\hat{f}_{\ell, t, s}, \tau_{n, t, s}$, $\mu_{\ell, j, t, s}^{+}$, and $\mu_{\ell, j, t, s}^{-}$, must satisfy the KKT conditions of the MI's lower-level problem.

Next, the KKT conditions can be replaced by disjunctive constraints [12]. For the MI's lower-level problem, these are

$$
\begin{aligned}
& -P_{s}\left(\tau_{n_{\ell}^{-}, t, s}-\tau_{n_{\ell}^{+}, t, s}\right)+\kappa_{\ell, t, s}=0, \forall \ell, t, s \\
& \psi_{\ell, j, t, s}+\mu_{\ell, j, t, s}^{+}-\mu_{\ell, j, t, s}^{-}-\kappa_{\ell, t, s}=0, \forall \ell, t, s, \forall j \in \mathcal{J}_{\ell} \\
& -\sum_{\ell \in \mathcal{L}_{n}^{+}} \sum_{j \in \mathcal{J}_{\ell}} B_{\ell, j}\left(\psi_{\ell, j, t, s}-\psi_{\ell, j, t, s}^{a u x}\right) \\
& \quad+\sum_{\ell \in \mathcal{L}_{n}^{-}} \sum_{j \in \mathcal{J}_{\ell}} B_{\ell, j}\left(\psi_{\ell, j, t, s}\right. \\
& \left.-\psi_{\ell, j, t, s}^{a u x}\right)+S_{n} \xi_{n, t, s}=0, \forall n, t, s \\
& -u_{\ell, j} M^{d} \leq \psi_{\ell, j, t, s}-\psi_{\ell, j, t, s}^{a u x} \leq u_{\ell, j} M^{d}, \\
& \forall \ell, t, s, \forall j \in \mathcal{J}_{\ell} \\
& -\left(1-u_{\ell, j}\right) M^{d} \leq \psi_{\ell, j, t, s}^{a u x} \leq\left(1-u_{\ell, j}\right) M^{d}, \\
& \forall \ell, t, s, \forall j \in \mathcal{J}_{\ell} \\
& -K_{\ell, j}^{a r c} u_{\ell, j} \leq f_{\ell, j, t, s} \leq K_{\ell, j}^{a r c} u_{\ell, j}, \forall \ell, t, s, \forall j \in \mathcal{J}_{\ell} \\
& -\left(1-u_{\ell, j}\right) M^{f l o w} \leq f_{\ell, j, t, s}-B_{\ell, j}\left(d_{n_{\ell}^{+}, t, s}-d_{n_{\ell}^{-}, t, s}\right) \\
& \leq\left(1-u_{\ell, j}\right) M^{f l o w}, \forall \ell, t, s, \forall j \in \mathcal{J}_{\ell} \\
& 0 \leq K_{\ell, j}^{a r c}-f_{\ell, j, t, s} \leq M^{a r c} w_{\ell, j, t, s}^{a r c}, \forall \ell, t, s, \forall j \in \mathcal{J}_{\ell} \\
& 0 \leq \mu_{\ell, j, t, s}^{+} \leq M^{a r c^{+}}\left(1-w_{\ell, j, t, s}^{a r c+}\right), \forall \ell, t, s, \forall j \in \mathcal{J}_{\ell} \\
& 0 \leq K_{\ell, j}^{a r c}+f_{\ell, j, t, s} \leq M^{a r c^{-}} w_{\ell, j, t, s}^{a r c}, \forall \ell, t, s, \forall j \in \mathcal{J}_{\ell}(4) \\
& 0 \leq \mu_{\ell, j, t, s}^{-} \leq M^{a r c^{-}}\left(1-w_{\ell, j, t, s}^{a r c}\right), \forall \ell, t, s, \forall j \in \mathcal{J}_{\ell}
\end{aligned}
$$




$$
\begin{aligned}
& \hat{f}_{\ell, t, s}-\sum_{j \in \mathcal{J}_{\ell}} f_{\ell, j, t, s}=0, \forall \ell, t, s \\
& S_{n} d_{n, t, s}=0, \forall n, t, s \\
& u_{\ell, j} \in\{0,1\}, \forall \ell, \forall j \in \mathcal{J}_{\ell}, \hat{f}_{\ell, t, s} \text { u.r.s., } \forall \ell, t, s \\
& f_{\ell, j, t, s} \text { u.r.s., } \forall \ell, t, s, \forall j \in \mathcal{J}_{\ell}, d_{n, t, s} \text { u.r.s., } \forall n, t, s \\
& \psi_{\ell, j, t, s} \text { u.r.s., } \psi_{\ell, j, t, s}^{a u x} \text { u.r.s., } \forall \ell, t, s, \forall j \in \mathcal{J}_{\ell} \\
& \kappa_{\ell, t, s} \text { u.r.s., } \forall \ell, t, s, \xi_{n, t, s} \text { u.r.s., } \forall n, t, s \\
& w_{\ell, j, t, s}^{a r c^{+}}, \in\{0,1\}, w_{\ell, j, t, s}^{a r c} \in\{0,1\}, \forall \ell, t, s, \forall j \in \mathcal{J}_{\ell} \\
& \tau_{n, t, s} \text { u.r.s., } \forall n, t, s .
\end{aligned}
$$

For conventional producer $i \in \mathcal{I}^{\mathrm{c}}$, they are

$$
\begin{aligned}
& 0 \leq-P_{s}\left(A_{n}^{i n t}-A_{n}^{s l p}\left(\left(1+\sum_{i^{\prime} \in \mathcal{I} \backslash\{i\}} \Theta_{i, i^{\prime}, n}\right) q_{n, i, t, s}^{\text {sell }}\right.\right. \\
& \left.\left.+\sum_{i^{\prime} \in \mathcal{I}} q_{n, i^{\prime}, t, s}^{\text {sell }}\right)-\tau_{n, t, s}\right)+\theta_{i, t, s} \leq M^{\text {sell }} w_{n, i, t, s}^{\text {sell }}, \\
& \forall n, t, s \\
& 0 \leq q_{n, i, t, s}^{\text {sell }} \leq M^{\text {sell }}\left(1-w_{n, i, t, s}^{\text {sell }}\right) \forall n, t, s \\
& 0 \leq P_{s}\left(C_{n, i}^{\text {prod }}-\tau_{n, t, s}+R v_{t, s}\right)+\lambda_{n, i, t, s}^{\mathrm{c}}-\theta_{i, t, s} \\
& \leq M^{\text {prod }} w_{n, i, t, s}^{\text {prod }}, \forall n, t, s \\
& 0 \leq q_{n, i, t, s}^{\text {prod }} \leq M^{\text {prod }}\left(1-w_{n, i, t, s}^{\text {prod }}\right), \forall n, t, s \\
& 0 \leq C_{n, i}^{i n v}-\sum_{s \in \mathcal{S}} \sum_{t \in \mathcal{T}} \lambda_{n, i, t, s}^{\mathrm{c}} \leq M^{i n v} w_{n, i}^{i n v}, \forall n \\
& 0 \leq g_{n, i}^{i n v} \leq M^{i n v}\left(1-w_{n, i}^{i n v}\right), \forall n \\
& 0 \leq K_{n, i}^{\text {prod }}+g_{n, i}^{\text {inv }}-q_{n, i, t, s}^{\text {prod }} \leq M^{\lambda^{\mathrm{c}}} w_{n, i, t, s}^{\lambda^{\mathrm{c}}}, \forall n, t, s \\
& 0 \leq \lambda_{n, i, t, s}^{\mathrm{c}} \leq M^{\lambda^{\mathrm{c}}}\left(1-w_{n, i, t, s}^{\lambda^{\mathrm{c}}}\right), \forall n, t, s \\
& \sum_{n \in \mathcal{N}} q_{n, i, t, s}^{\text {prod }}-\sum_{n \in \mathcal{N}} q_{n, i, t, s}^{s e l l}=0, \forall n, t, s \\
& \theta_{i, t, s} \text { u.r.s., } \forall t, s \\
& w_{n, i, t, s}^{\text {prod }}, w_{n, i, t, s}^{\text {sell }}, w_{n, i, t, s}^{\lambda^{\mathrm{c}}} \in\{0,1\}, \forall n, t, s \\
& w_{n, i}^{i n v} \in\{0,1\}, \forall n .
\end{aligned}
$$

Analogously, for wind producer $i \in \mathcal{I}^{\mathrm{w}}$, the disjunctive constraints are the same as in (52)-(63) with the following replacements for (54), (56), (58), and (59):

$$
\begin{aligned}
0 \leq & -P_{s}\left(\tau_{n, t, s}+(1-R) v_{t, s}\right)+\lambda_{n, i, t, s}^{\mathrm{w}}-\theta_{i, t, s} \\
\leq & M^{\text {prod }} w_{n, i, t, s}^{\text {prod }}, \forall n, t, s \\
0 \leq & C_{n, i}^{i n v}-\sum_{s \in \mathcal{S}} \sum_{t \in \mathcal{T}} \lambda_{n, i, t, s}^{\mathrm{w}} E_{n, t, s} \leq M^{i n v} w_{n, i}^{i n v}, \forall n \\
& q_{n, i, t, s}^{\text {prod }}-E_{n, t, s}\left(K_{n, i}^{\text {prod }}+g_{n, i}^{i n v}\right)=0, \forall n, t, s \\
& \lambda_{n, i, t, s}^{\mathrm{w}} \text { u.r.s., } \forall n, t, s .
\end{aligned}
$$

Finally, the market-clearing and RPS constraints become

$$
\begin{aligned}
& \sum_{i \in \mathcal{I}} q_{n, i, t, s}^{\text {sell }}-\sum_{i \in \mathcal{I}} q_{n, i, t, s}^{\text {prod }}+\sum_{\ell \in \mathcal{L}_{n}^{+}} \hat{f}_{\ell, t, s}-\sum_{\ell \in \mathcal{L}_{n}^{-}} \hat{f}_{\ell, t, s}=0, \\
& \forall n, t, s \\
0 \leq & \sum_{n \in \mathcal{N}} \sum_{i \in \mathcal{I}^{\mathrm{w}}} q_{n, i, t, s}^{\text {prod }}-R \sum_{n \in \mathcal{N}} \sum_{i \in \mathcal{I}} q_{n, i, t, s}^{\text {prod }} \leq M^{v} w_{t, s}^{v} \\
& \forall t, s \\
0 \leq & v_{t, s} \leq M^{v}\left(1-w_{t, s}^{v}\right), \forall t, s
\end{aligned}
$$

$$
\begin{aligned}
& \tau_{n, t, s} \text { u.r.s., } \forall n, t, s \\
& w_{t, s}^{v} \in\{0,1\}, \forall t, s .
\end{aligned}
$$

Hence, the MILP for the MI is

$$
\max _{\left\{u_{\ell, j}\right\} \cup \Gamma \cup \Gamma_{i} \cup-10000 f_{i l}^{M I} \cup \Phi^{M I}}
$$

s.t. (2)-(3); (38)-(51); (52)-(53), (55), (57),

(60)-(63) $\forall i \in \mathcal{I} ;(54),(56),(58),(59) \forall i \in \mathcal{I}^{\mathrm{c}}$;

(64)-(67) $\forall i \in \mathcal{I}^{\mathrm{w}} ;(68)-(72)$

where $\Phi^{M I}=\left\{w_{n, i, t, s}^{\text {prod }}, w_{n, i, t, s}^{\text {sell }}, w_{n, i}^{i n v}, w_{n, i, t, s}^{\lambda^{\mathrm{c}}}, w_{n, i, t, s}^{\lambda^{\mathrm{w}}}\right.$, $\left.w_{\ell, j, t, s}^{a r c^{+}}, w_{\ell, j, t, s}^{a r c^{-}}, w_{t, s}^{v}, \psi_{\ell, j, t, s}^{a u x}\right\}$.

Since the TSO's objective function (35) is quadratic, we directly use disjunctive constraints to formulate its MIQP:

$$
\max _{\left\{u_{\ell, j}\right\} \cup \Gamma \cup \Gamma_{i} \cup-10000 \text { fil }}{ }^{T S O} \cup \Phi^{T S O}
$$

$$
\begin{aligned}
& \text { s.t. }(2)-(3) ;(8)-(10) ;(43)-(44) ;(52)-(53),(55),(57) \text {, } \\
& \quad(60)-(63) \forall i \in \mathcal{I} ;(54),(56),(58),(59) \forall i \in \mathcal{I}^{\mathrm{c}} ; \\
& \quad(64)-(67) \forall i \in \mathcal{I}^{\mathrm{w}} ;(68)-(72)
\end{aligned}
$$

where $\Phi^{T S O}=\left\{w_{n, i, t, s}^{\text {prod }}, w_{n, i, t, s}^{\text {sell }}, w_{n, i}^{i n v}, w_{n, i, t, s}^{\lambda^{\mathrm{c}}}, w_{n, i, t, s}^{\lambda^{\mathrm{w}}}\right.$, $\left.w_{t, s}^{v}\right\}$. Likewise, the CP's MINLP may be re-formulated as an MIQP:

$$
\begin{aligned}
\max _{\left\{u_{\ell, j}\right\} \cup \Gamma \cup \Gamma_{i}} & (35) \\
\text { s.t. } & (2)-(3) ;(8)-(10) ;(20) \forall i \in \mathcal{I}^{\mathrm{c}} ; \\
& (21)-(22) \forall i \in \mathcal{I} ;(29) \forall i \in \mathcal{I}^{\mathrm{w}} ; \\
& (33) ;(36) ;(43)-(44) .
\end{aligned}
$$

\section{ACKNOWLEDGMENT}

The authors would like to thank S.-E. Fleten for feedback on an earlier version of the paper. Comments from attendees of CMS 2013, EEM 2013, INFORMS 2013, IFORS 2014, and OR56 conferences as well as seminars at DTU, LSE, and Høgskolen i Sogn og Fjordane have also been helpful. Finally, suggestions from the handling editor and three anonymous referees have improved the paper. All remaining errors are the authors' own.

\section{REFERENCES}

[1] L. S. Hyman, "Restructuring electricity policy and financial models," Energy Econ., vol. 32, no. 4, pp. 751-757, Jul. 2010.

[2] R. B. Wilson, "Architecture of power markets," Econometrica, vol. 70, no. 4, pp. 1299-1340, Jul. 2002.

[3] B. F. Hobbs and U. Helman, "Complementarity-based equilibrium modeling for electric power markets," in Modeling Prices in Competitive Electricity Markets, D. W. Bunn, Ed., 2nd ed. New York, NY, USA: Wiley, 2004.

[4] "Regulation (EC) No 1228/2003 of the European Parliament and of the Council of 26 June 2003 on Conditions for Access to the Network for Cross-Border Exchanges in Electricity," in Eur. Commission, Brussels, Belgium [Online]. Available: http://eur-lex.europa.eu/legal-content/en/TXT/?uri=CELEX:32003R1228

[5] "A Policy Framework for Climate and Energy in the Period from 2020 to 2030, $\operatorname{COM}(2014)$ 015," in Eur. Commission, Brussels, Belgium, 2014 [Online]. Available: http://eur-lex.europa.eu/legal-content/EN/TXT/?uri=CELEX:52014DC0015 
[6] F. Kunz, "Improving congestion management: How to facilitate the integration of renewable generation in Germany," Energy J., vol. 34, no. 4, pp. 55-78, Oct./Dec. 2013.

[7] B. F. Hobbs, "Optimization methods for electric utility resource planning," Eur. J. Oper. Res., vol. 83, no. 1, pp. 1-20, May 1995.

[8] S. A. Gabriel, A. J. Conejo, J. D. Fuller, B. F. Hobbs, and C. Ruiz, Complementarity Modeling in Energy Markets. New York, NY, USA: Springer, 2012.

[9] C. Ruiz, A. J. Conejo, J. D. Fuller, S. A. Gabriel, and B. F. Hobbs, "A tutorial review of complementarity models for decision-making in energy markets," EURO J. Dec. Proc., to be published.

[10] B. F. Hobbs, "Linear complementarity models of Nash-Cournot competition in bilateral and poolco power markets," IEEE Trans. Power Syst., vol. 16, no. 2, pp. 194-202, May 2001.

[11] C. Ruiz and A. J. Conejo, "Pool strategy of a producer with endogenous formation of locational marginal prices," IEEE Trans. Power Syst., vol. 24, no. 4, pp. 1855-1866, Nov. 2009

[12] J. Fortuny-Amat and B. McCarl, "A representation and economic interpretation of a two-level programming problem," J. Oper. Res. Soc., vol. 32, no. 9, pp. 783-792, Sep. 1981.

[13] S. Wogrin, E. Centeno, and J. Barquín, "Generation capacity expansion in liberalized electricity markets: a Stochastic MPEC approach," IEEE Trans. Power Syst., vol. 26, no. 4, pp. 2526-2532, Nov. 2011.

[14] L. P. Garcés, A. J. Conejo, R. García-Bertrand, and R. Romero, "A bilevel approach to transmission expansion planning within a market environment," IEEE Trans. Power Syst., vol. 24, no. 3, pp. 1513-1522, Aug. 2009.

[15] L. Baringo and A. J. Conejo, "Transmission and wind power investment," IEEE Trans. Power Syst., vol. 27, no. 2, pp. 885-893, May 2012.

[16] D. J. Burke and M. J. O'Malley, "Maximum firm wind power connection to security constrained transmission networks," IEEE Trans. Power Syst., vol. 25, no. 2, pp. 749-759, May 2010.

[17] S. Wogrin, B. F. Hobbs, D. Ralph, E. Centeno, and J. Barquín, "Open versus closed loop capacity equilibria in electricity markets under perfect and oligopolistic competition," Math. Prog., vol. 140, no. 2, pp. 295-322, Sep. 2013.

[18] D. M. Kreps and J. A. Scheinkman, "Quantity precommitment and Bertrand competition yield Cournot outcomes," Bell J. Econ., vol. 14, no. 2, pp. 326-337, 1983.

[19] E. Sauma and S. S. Oren, "Proactive planning and valuation of transmission investments in restructured electricity markets," J. Reg. Econ., vol. 30, pp. 261-290, Dec. 2006.

[20] E. Sauma and S. S. Oren, "Economic criteria for planning transmission investment in restructured electricity markets," IEEE Trans. Power Syst., vol. 22, no. 4, pp. 1394-1405, Nov. 2007.

[21] W. Hogan, "Contract networks for electric power transmission," $J$ Reg. Econ., vol. 4, no. 3, pp. 211-242, Sep. 1992.

[22] P. Joskow and J. Tirole, "Merchant transmission investment," J. Ind. Econ., vol. 53, no. 2, pp. 233-264, Jun. 2005.
[23] T. Kristiansen and J. Rosellón, "Merchant electricity transmission expansion: A European case study," in DIW Discussion Papers, Berlin, Germany, 2010.

[24] E. Bartholomew, A. S. Siddiqui, C. Marnay, and S. S. Oren, "The New York transmission congestion contract market: Is it truly working efficiently?," Electricity J., vol. 16, no. 9, pp. 14-24, Nov. 2003.

[25] M. Tanaka and Y. Chen, "Market power in renewable portfolio standards," Energy Econ., vol. 39, pp. 187-196, Sep. 2013.

[26] T. Limpaitoon, Y. Chen, and S. S. Oren, "The impact of imperfect competition in emission permits trading on oligopolistic electricity markets," Energy J., vol. 35, no. 3, pp. 145-166, 2014.

[27] F. Murphy and Y. Smeers, "On the impact of forward markets on investments in oligopolistic markets with reference to electricity," Oper. Res., vol. 58, no. 3, pp. 512-528, 2010

[28] M. Reta-Hernández, "Transmission line parameters," in Electric Power Generation, Transmission, and Distribution, L. L. Grigsby, Ed., 3rd ed. Boca Raton, FL, USA: CRC Press, 2012.

Lajos Maurovich-Horvat received the B.Sc. and M.Res. degrees from University College London, U.K., in economics-statistics and financial computing, respectively. He is currently pursuing the Ph.D. degree in the Department of Statistical Science at University College London.

His research interests are in decision analysis for sustainable energy technologies via stochastic optimization.

Trine K. Boomsma received the B.S., M.S., and Ph.D. degrees from Aarhus University, Denmark, all in mathematics-economics with particular focus on operations research.

She is currently an Associate Professor in the Department of Mathematical Sciences, University of Copenhagen, Denmark. Her research interests evolve around applications of operations research to energy and electricity problems. This includes stochastic programming and real options analysis for operations scheduling, bidding problems, and investment assessment

Afzal S. Siddiqui received the B.S. degree from Columbia University, New York, NY, USA, and the M.S. and Ph.D. degrees from the University of California, Berkeley, CA, USA, all in industrial engineering and operations research. $\mathrm{He}$ is a Senior Lecturer in the Department of Statistical Science at University College London and a Professor in the Department of Computer and Systems Sciences at Stockholm University. His research interests lie in investment and operational analysis with applications to the energy sector. In particular, he focuses on distributed generation investment and operation under uncertainty, real options analysis of technology adoption, and strategic interactions in capacity expansion. 